\title{
Intervalos de corte de pasto Saboya (Panicum máximum Jacq.), sobre rendimiento de materia seca y composición química de su ensilaje
} grass (Panicum máximum Jacq.) and its silage chemical composition

\author{
Katy Derichs ${ }^{1}$, Jorge Mosquera ${ }^{2}$, Lenin Javier Ron-Garrido ${ }^{3}$, \\ Byron Puga-Torres ${ }^{4}$, Francisco De la Cueva ${ }^{5}$
}

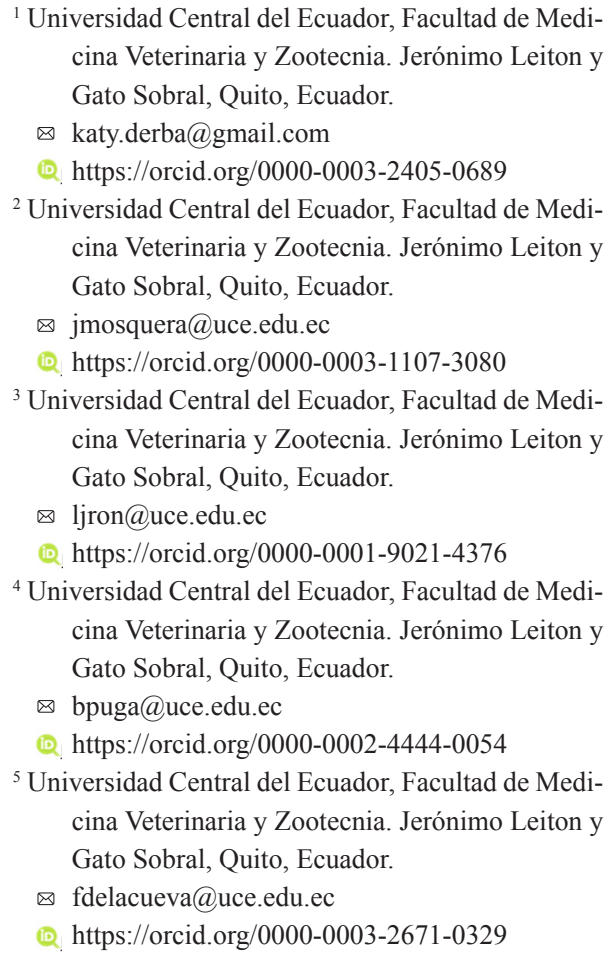

*Autor de correspondencia: bpuga@uce.edu.ec

\section{SIEMBRA}

https://revistadigital.uce.edu.ec/index.php/SIEMBRA ISSN-e: $2477-8850$

ISSN: $1390-8928$

Periodicidad: semestral

vol. 8, núm. 2, 2021

siembra.fag@uce.edu.ec

DOI: https://doi.org/10.29166/siembra.v8i2.2506

Esta obra está bajo una licencia internacional Creative Commons Atribución-NoComercial

\section{Resumen}

Durante la época seca en el norte del litoral ecuatoriano, se presenta escasez de pasto aspecto que requiere resolverse. El objetivo de la presente investigación fue evaluar el efecto de diferentes intervalos de corte sobre el rendimiento de biomasa seca, composición química y calidad nutricional de ensilaje del pasto saboya (Panicum máximum Jacq.). Durante la transición de época lluviosa a seca, en la parroquia Viche de la provincia de Esmeraldas en Ecuador, se evaluó la biomasa seca, características organolépticas, composición química y de las paredes celulares del ensilaje de pasto Saboya cortado a edades de 13, 20, 27, 34 y 41 días. Los tratamientos se implementaron en un Diseño Completamente al Azar para las características del pasto y composición química del ensilaje, y en un diseño de Bloques Completos al Azar para las características organolépticas del ensilaje. El análisis estadístico se basó en el Análisis de Varianza y comparación múltiple de medias entre tratamientos usando Tukey al $5 \%$. En la investigación se determinó que las características organolépticas de los ensilajes a los 20 y 34 días fueron de mejor calidad. En el análisis bromatológico se determinó que el ensilaje a los 27 días contiene una media de $8,25 \%$ de proteína bruta, que es estadísticamente mayor que todos los tratamientos evaluados, seguido por el ensilaje a los 41 días con 5,53\% de proteína bruta. El rendimiento de materia seca anual varió de 9,74 a 29,98 t ha $^{-1}$. El ensilaje preparado a los 27 días presentó los mejores valores nutricionales, mientras que el mejor rendimiento se presentó a los 41 días. Ningún tratamiento alcanzó un nivel óptimo de fermentación $(\mathrm{pH}$, lignificación relación tallo/hoja).

Palabras clave: composición química, corte de pasto, ensilaje, Saboya.

\begin{abstract}
During the dry season in the north of the Ecuadorian coast, there is a grass shortage, aspect that need to be solved. The objective of this research was to evaluate the effect of different cutting intervals on the dry biomass yield, chemical composition and nutritional quality of savoy grass (Panicum maximum Jacq.). During the rainy season transition to dry, in the Viche locality, in the province of Esmeraldas in Ecuador, the dry biomass, organoleptic characteristics, chemical and cell wall composition of
\end{abstract}


savory grass silage cut at ages of 13, 20, 27, 34 and 41 days were evaluated. Treatments were set up in a Complete Random Design for the pasture characteristics and chemical composition of the silage, and in a Randomized Complete Block Design for the organoleptic characteristics of the silage. The statistical analysis was based on the Analysis of Variance and the multiple mean comparisons between treatments with Tukey at $5 \%$. In this research, the quality of silage organoleptic characteristics was higher at 20 and 34 days. Bromatological analyses showed a content of $8.25 \%$ of crude protein at 27-day silage preparation, being the highest of all treatments, followed by silage preparation at 41 -day with $5.53 \%$ of crude protein. The annual dry matter yield ranged from 9.74 to $29.98 \mathrm{t} \mathrm{ha}^{-1}$. Silage prepared at 27 days had the highest nutritional values, while yield was higher at 41 days. None of the treatments reached an optimal level of fermentation ( $\mathrm{pH}$, lignification, stem/leaf ratio).

Keywords: chemical composition, grass cutting, silage, savoy grass.

\section{Introducción}

La mayor producción de leche cruda de vaca en Ecuador se desarrolla en la región Interandina, especialmente en la provincia de Pichincha, donde se produce más del $13 \%$ de la producción nacional, cuya mayor cuenca lechera es el cantón Mejía y donde se han realizado la mayoría de estudios y avances tecnológicos, descuidando indirectamente las zonas tropicales del país (Instituto Nacional de Estadísticas y Censos [INEC], 2014, 2021; Venegas, 2017). En la región costera, el pasto Saboya (Panicum maximum Jacq.) es el forraje predominante, teniendo un excedente en la época de lluvias y una escasez en la época seca, por lo que existe una subutilización del pasto en la primera (Núñez-Delgado et al., 2019). Así mismo, analizando la ganancia diaria de peso (GDP) de vaquillonas menores de 18 meses, criadas en la provincia de Esmeraldas-Ecuador, se evidenció que ésta no supera los 400 gramos, lo que se traduce en un retraso en el crecimiento y, por ende, en la edad en la que se logra el peso ideal para la primera monta, provocando que las vaquillonas no lleguen al primer servicio a los 18 meses, lo que genera atrofia de la glándula mamaria; esto sumado a la escasez de alimento durante la época seca, entre junio y agosto, se traduce en sistemas productivos poco eficientes (Del Pezo, 2015; Machado, 2016; Villota González, 2012).

Para contrarrestar esta situación, existen alternativas de conservación de forrajes como la henificación, la producción de harinas de pasto y el ensilaje (Batello, 2000; Mannetje, 2005). Este último es considerado una alternativa económica y práctica frente a la escasez de alimento a lo largo del año, pudiendo evitar pérdidas en la producción lechera, al ser una reserva de alimento (Batello, 2000; Del Pezo, 2015; Machado, 2016) con una fuente importante de nutrientes, principalmente de fibra digestible y energía, siempre y cuando esté bien fermentado y conservado (Ohmomo et al., 2002; Queiroz et al., 2018). Sin embargo, la elaboración de ensilaje en la estación seca, no es una práctica común en el trópico ecuatoriano, debido a que sus pastos tienen alto contenido de humedad y bajos niveles de carbohidratos solubles, lo que impide el ensilaje (Nussio et al., 2002); igualmente, la fermentación de ensilajes tropicales no se traduce en considerables cantidades de ácido láctico, el cual es el conservante ideal (Parvin y Nishino, 2009).

El pasto Saboya o pasto Guinea pertenece al género Panicum, tanto en formas anuales como perennes, y es originario de África (Pessim et al., 2015). Tiene un alto rendimiento de forraje (entre 2 y $3 \mathrm{~kg} \mathrm{~m}^{-2}$ ) de buena calidad y buena aceptación por parte de los animales, teniendo un promedio de producción por hectárea de 46,28 kg de materia seca (MS) hectárea ${ }^{-1}$ (ha) día ${ }^{-1}$ en época lluviosa, 18,42 $\mathrm{kg} \mathrm{MS}^{-1} \mathrm{ha}^{-1}$ día $^{-1}$ en el inicio de las lluvias y solamente $8,16 \mathrm{~kg} \mathrm{MS}^{-1} \mathrm{ha}^{-1}$ día $^{-1}$ en la época seca (Núñez Delgado et al., 2019). Estudios realizados por Parvin y Nishino (2009) indican que después de varias semanas del ensilado de Saboya el nivel de ácido láctico disminuía, mientras que el nivel de ácido acético aumentaba, lo que sugiere que la evaluación de este pasto ensilado podría verse influenciada por la materia seca del cultivo y el período de almacenamiento; sin embargo, se observa una mejora significativa cuando se usa melaza, la cual suple a los carbohidratos fácilmente fermentables, necesarios para el proceso. Existen varios estudios de ensilaje de pastos tropicales, pero ninguno de ellos con pastos de edades menores a 35 días, debido a bajos niveles de MS, aunque se establece que el pasto Saboya, alcanza hasta el $10 \%$ de proteína cruda (PC) a los 10 días de edad, mientras que a los 45 días disminuye al $8 \%$ y baja al $6 \%$ a los 60 días (Rodríguez-López, 2009; Wascheck et al., 2008). Respecto a la energía metabolizable, también existen diferencias en relación con la edad del pasto, siendo los mejores valores a los 30 días, en donde alcanza 10,09 megajulios (Mj) $\mathrm{kg}^{-1} \mathrm{MS}$ (período lluvioso) y $10,17 \mathrm{Mj} \mathrm{kg}^{-1} \mathrm{MS}^{-}$ (período poco lluvioso); en cambio a los 105 días se encuentra los valores más bajos, siendo de 7,45 $\mathrm{Mj} \mathrm{kg}^{-1}$ 
MS (período lluvioso) y 7,61 $\mathrm{Mj} \mathrm{kg}^{-1} \mathrm{MS}$ (período poco lluvioso) (Verdecia et al., 2008), lo cual podría deberse a que las condiciones de temperatura y la disponibilidad de agua pueden afectar la biomasa de producción de Saboya (Olivera Viciedo et al., 2019), siendo la temperatura elevada la causante de alterar el contenido de aminoácidos y metabolitos secundarios (Wedow et al., 2019). Actualmente existen varios estudios que proporcionan mecanismos bioinformáticos para una mejor selección genómica que lleve a mejoras en la composición nutricional de este pasto (Lara et al., 2019).

Según la encuesta de superficie y producción agropecuaria (ESPAC) del año 2018, en Ecuador se cultivaron un total de 2.382.448 ha, siendo el pasto Saboya el más usado, pues ocupó el 42,9 \% de la superficie total plantada a nivel nacional (INEC, 2019), sobre todo en la Costa y en menor cantidad en la región oriental (INEC, 2014). Esta variedad de pasto puede ser ensilado y está al alcance de pequeños, medianos y grandes productores, haciendo posible su conservación sin necesidad de invertir en nuevos pastos (Montenegro, 2019). En el Ecuador se ha investigado el ensilaje de pasto Saboya de 45 y 60 días con inclusión de contenido ruminal y se compararon los periodos de ensilaje de 21 y 35 días, obteniéndose mejores resultados a los 21 días (González Albarracín, 2013). Por esta razón la presente investigación tuvo como objetivo determinar la calidad nutricional, a través de un análisis bromatológico, del ensilaje de pasto Saboya a diferentes edades de corte: 13, 20, 27, 34 y 41 días, a fin de encontrar la edad óptima de la pastura para el proceso de ensilaje, que permita suplir la deficiencia de pasto durante la época seca en la provincia de Esmeraldas, en el litoral norte de Ecuador.

\section{Materiales y métodos}

El estudio se llevó a cabo en la hacienda "El Triunfo", ubicada en el km 43 de la vía Quinindé, parroquia Viche, en un área asignada de $980 \mathrm{~m}^{2}$, dentro de ésta el área de estudio fue de $180 \mathrm{~m}^{2}$. La misma pertenece a la provincia costera de Esmeraldas. Las características ambientales fueron medidas por la estación meteorológica de la propiedad (Eurocrhron Touch Weather Center W CDF77), y presentaron una temperatura entre 20,8 y $30^{\circ} \mathrm{C}$, humedad entre 45 y $99 \%$, un pH del suelo de 5,6 (ligeramente ácido). La altitud máxima de la parroquia es de 540 metros sobre el nivel del mar.

El estudio es de tipo experimental, mediante un diseño completamente al azar (DCA) para la comparación de medias de las características del pasto y de la composición química del ensilaje y un diseño en bloques completos al azar (DBCA) para el análisis de las características organolépticas del ensilaje, donde los bloques fueron los evaluadores. Un total de 8 personas, seleccionadas al azar, calificaron las características organolépticas del ensilaje, quienes no recibieron capacitación previa sobre el procedimiento de calificación con el formato implementado, el mismo que consistió en un cuestionario establecido.

Se establecieron 20 parcelas de $9 \mathrm{~m}^{2}(3 \times 3)$, para 5 tratamientos diferentes con 4 repeticiones cada uno. Se realizaron cortes de uniformización en las diferentes parcelas de pasto Saboya, con 7 días de diferencia entre tratamientos, con la finalidad de que puedan ser cortados y ensilados el mismo día. Los cortes se realizaron con tijeras de jardinería, a $20 \mathrm{~cm}$ del suelo, a los 13, 20, 27, 34 y 41 días de edad. Luego del corte se midió el rendimiento de materia verde (MV) para el posterior análisis de rendimiento de MS, así como la relación hoja/tallo $(\mathrm{H} / \mathrm{T})$.

Para el cálculo de MV, se multiplica por 40 el valor de peso fresco de forraje obtenido en el corte. Mientras que la MS se determinó por gravimetría, expresándose en $\mathrm{kg} \mathrm{ha}^{-1}$. Para determinar la relación $\mathrm{H} / \mathrm{T}$, se tomaron muestras de la MV homogenizada de cada unidad experimental. En cada muestra se apartaron las hojas de los tallos, se pesaron por separado y se dividió el peso de las hojas para el peso de los tallos.

Los microsilos se fabricaron en tubo PVC de $5,5 \mathrm{~cm}$ de diámetro y $55 \mathrm{~cm}$ de altura. De cada parcela se tomaron $3 \mathrm{~kg}$ de pasto secado al sol por dos horas, a una temperatura ambiental de entre 27,4 y $32,4{ }^{\circ} \mathrm{C}$, picado en partículas de 1-3 cm, para ser colocado en cada microsilo, los cuales se sellaron luego de la aplicación de presión. Los silos se mantuvieron cerrados por 32 días.

Los análisis bromatológicos se realizaron en el Laboratorio de Nutrición Animal de la Facultad de Ciencias Agrícolas de la Universidad Central del Ecuador, donde se analizaron las características organolépticas con la ayuda de un sistema de puntuación (Tabla 1) creado por la Sociedad Alemana de Agricultura (DLG), con la finalidad de clasificar al ensilaje como muy bueno, bueno, regular, malo o muy malo, con base en la puntuación final obtenida. 
Tabla 1. Sistema de puntuación de las características organolépticas del ensilado

Table 1. Scoring system for the silage organoleptic characteristics

\begin{tabular}{|c|c|c|}
\hline \multirow{26}{*}{ Olor } & Ácido butírico (olor a mantequilla rancia) & \\
\hline & Imperceptible & 0 \\
\hline & Leve, se impregna en los dedos & 2 \\
\hline & Se percibe leve sin la necesidad de tocar el ensilaje & 3 \\
\hline & A $1 \mathrm{~m}$ de distancia se percibe claramente el olor & 5 \\
\hline & Muy perceptible a más de 1 metro de distancia & 7 \\
\hline & Ácido acético (olor a vinagre) & \\
\hline & Imperceptible & 0 \\
\hline & Poco perceptible & 1 \\
\hline & Claramente perceptible & 2 \\
\hline & Muy perceptible, desagradable e incómodo & 4 \\
\hline & Olor a quemado & \\
\hline & Imperceptible & 0 \\
\hline & Tostado, ligero y agradable & 1 \\
\hline & Tostado, ligeramente ahumado & 2 \\
\hline & Fuertemente quemado & 4 \\
\hline & Levaduras (fermentado) & \\
\hline & Imperceptible & 0 \\
\hline & Poco perceptible & 1 \\
\hline & Claramente perceptible & 2 \\
\hline & Muy perceptible & 4 \\
\hline & Hongos (olor a humedad) & \\
\hline & Imperceptible & 0 \\
\hline & Poco perceptible & 3 \\
\hline & Claramente perceptible & 5 \\
\hline & Muy perceptible & 7 \\
\hline \multirow{13}{*}{ Color } & Coloración marrón & \\
\hline & Normal & 0 \\
\hline & Más marrón de lo normal & 1 \\
\hline & Claramente marrón & 2 \\
\hline & Marrón oscuro & 4 \\
\hline & Coloración amarillenta & \\
\hline & Normal & 0 \\
\hline & Más amarillento de lo normal & 1 \\
\hline & Claramente amarillento & 2 \\
\hline & Amarillo & 4 \\
\hline & Otras observaciones & \\
\hline & Verde fuerte, fuerte fermentación ácido-butírica & 7 \\
\hline & Moho visible (no administrar ensilaje) & 7 \\
\hline \multirow{5}{*}{ Descomposición } & Ninguna parte afectada & 0 \\
\hline & Bordes de las hojas ligeramente afectadas & 1 \\
\hline & Hojas claramente afectadas, untuosa & 2 \\
\hline & Hojas y tallos afectados, podrido, descompuesto & 4 \\
\hline & TOTAL & $\Sigma *$ \\
\hline
\end{tabular}

* Puntuación: 0-1 Muy Bueno; 2-3 Bueno; 4-5 Regular; 6-8 Malo; >8 Muy malo

Fuente: Junges (2010); Nussbaum et al. (2004) 
La composición química se estableció por medio de un análisis proximal, para las paredes celulares por el método de Van Soest (usando una solución de sulfato lauril sódico en un $\mathrm{pH}$ neutro) y el pH por potenciometría (Junges, 2010; Nussbaum et al., 2004).

Una vez realizada la corrección de datos se efectuó la prueba de comparaciones de medias a través de un análisis de varianza (ANOVA), mientras que las comparaciones múltiples se realizaron mediante la prueba de Tukey con $5 \%$ de significancia. El procesamiento de datos se realizó a través del programa estadístico "R" (Versión R 3.3.3) y los paquetes adicionales "agricolae", "gvlma", "psych" y "MASS".

\section{Resultados}

Se implementó una superficie de corrección de datos (Figura 1), ya que se encontró que hay pérdida de humedad del pasto fresco debido a las drásticas condiciones ambientales presentes a las horas del corte, por lo que esta pérdida se presenta en función de la cantidad de pasto cosechado, el cual sufre mayor pérdida de humedad mientras menor es la cantidad de pasto cosechado y la temperatura ambiental presente; de esta forma se está sobreestimando su MS. El rendimiento de MV (secada al sol por 2 horas - MS) y la relación H/T del pasto, se detallan en la Tabla 2.

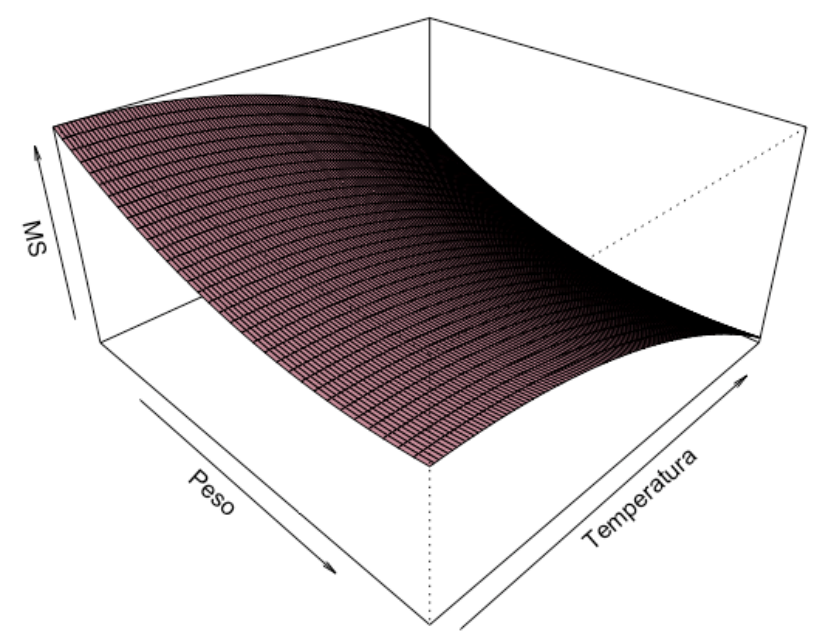

Figura 1. Superficie de corrección de datos.

Figure 1. Data correction area.

Tabla 2. Rendimiento de biomasa verde vs. relación H/T del pasto Saboya

Table 2. Green biomass yield vs. leaf/stem ratio of savoy grass

\begin{tabular}{|c|c|c|c|c|}
\hline Edad (días) & $\begin{array}{l}\text { MS } \\
(\%)\end{array}$ & $\begin{array}{c}\text { MS ha } \text { corte }^{-1} \\
(\mathrm{tm})\end{array}$ & $\begin{array}{c}\text { MS ha' } \text { año }^{-1} \\
\text { (tm) }\end{array}$ & $\begin{array}{c}\text { Relación } \\
\text { H/T }\end{array}$ \\
\hline 13 & $20,22 \mathrm{c}$ & $0,35 \mathrm{~d}$ & $9,74 \mathrm{e}$ & $1,58 \mathrm{~b}$ \\
\hline 20 & $23,47 \mathrm{bc}$ & $0,85 \mathrm{c}$ & $15,38 \mathrm{~d}$ & $1,62 \mathrm{~b}$ \\
\hline 27 & $25,73 \mathrm{ab}$ & $1,22 \mathrm{~b}$ & $16,54 \mathrm{c}$ & $3,36 \mathrm{a}$ \\
\hline 34 & $26,86 \mathrm{a}$ & $2,60 \mathrm{a}$ & $27,87 \mathrm{~b}$ & $2,11 \mathrm{~b}$ \\
\hline 41 & $29,09 \mathrm{a}$ & $3,37 \mathrm{a}$ & 29,98 a & $1,24 \mathrm{~b}$ \\
\hline$p$ & $0,000^{*}$ & $0,0001 *$ & $0,0005^{*}$ & $0,0002 *$ \\
\hline
\end{tabular}

*Significación estadística de $p$ al $5 \%$

Nota: Los resultados que tienen la misma letra no tienen diferencia significativa. 


\subsection{Rendimiento de materia seca y relación H/T del pasto Saboya}

El rendimiento ( $\mathrm{MS} \mathrm{ha}^{-1}$ corte $^{-1}$ ) estuvo determinado por la edad del pasto, aumentando aproximadamente 10 veces el peso, de 0,35 t ha ${ }^{-1} \operatorname{corte}^{-1}$ a los 13 días hasta 3,37 $\mathrm{t} \mathrm{ha}^{-1} \operatorname{corte}^{-1}$ a los 41 días (Tabla 2). Así mismo, la relación $\mathrm{H} / \mathrm{T}$ aumentó con la edad hasta los 27 días, donde alcanzó el valor máximo (3,36 \%) y disminuyó en edades de $34(2,11 \%)$ y $41(1,24 \%)$ días, presentando una diferencia estadística significativa entre el tratamiento de 27 días con respecto a los demás (Tabla 2).

\subsection{Análisis organoléptico del ensilaje de pasto Saboya (al momento del corte)}

El ANOVA determinó que las características organolépticas variaron de acuerdo con la edad en la que el pasto fue ensilado, excepto por "olor ácido acético" y "color amarillento", que no presentaron diferencias significativas. Sin embargo, estas diferencias no se reflejaron en la puntuación, por lo que, en general, todas las medias de los tratamientos comparten la misma calificación, excepto los olores a ácido butírico y levaduras (Tabla 3).

Tabla 3. Características organolépticas del ensilaje de pasto Saboya

Table 3. Organoleptic characteristics of savoy grass silage

\begin{tabular}{|c|c|c|c|c|c|c|c|c|}
\hline \multirow[b]{2}{*}{ Edad } & \multicolumn{5}{|c|}{ Olor } & \multicolumn{2}{|c|}{ Color } & \multirow{2}{*}{$\begin{array}{c}\text { Nivel de } \\
\text { descomposición }\end{array}$} \\
\hline & $\begin{array}{c}\text { Ácido } \\
\text { butírico }^{\mathrm{a}}\end{array}$ & $\begin{array}{c}\text { Ácido } \\
\text { acético }^{\text {b }}\end{array}$ & Quemado $^{\text {b }}$ & Levaduras $^{b}$ & Hongos $^{\mathrm{c}}$ & Marrón b & Amarillo $^{\mathrm{b}}$ & \\
\hline 13 & $2,64 \mathrm{~b}$ & 0,78 & $0,78 \mathrm{c}$ & $1,04 \mathrm{~b}$ & $1,70 \mathrm{~b}$ & $0,60 \mathrm{c}$ & 0,29 & $0,54 \mathrm{~b}$ \\
\hline 20 & $1,32 \mathrm{a}$ & 0,55 & $0,08 \mathrm{a}$ & $0,44 \mathrm{a}$ & $0,21 \mathrm{a}$ & $0,04 \mathrm{a}$ & 0,57 & $0,18 \mathrm{a}$ \\
\hline 27 & $2,14 \mathrm{ab}$ & 1,09 & $0,44 \mathrm{~b}$ & $0,56 \mathrm{a}$ & $0,43 \mathrm{a}$ & $0,35 \mathrm{~b}$ & 0,42 & $0,12 \mathrm{a}$ \\
\hline 34 & $1,57 \mathrm{a}$ & 0,84 & $0,25 \mathrm{a}$ & $0,46 \mathrm{a}$ & $0,44 \mathrm{a}$ & $0,16 \mathrm{ab}$ & 0,46 & $0,14 \mathrm{a}$ \\
\hline 41 & $2,57 \mathrm{~b}$ & 0,73 & $0,46 \mathrm{~b}$ & $0,86 \mathrm{~b}$ & $1,11 \mathrm{ab}$ & $0,08 \mathrm{a}$ & 0,43 & $0,39 \mathrm{~b}$ \\
\hline
\end{tabular}

Nota: Los resultados que tienen la misma letra no tienen diferencia significativa.

Escalas de valoración: ${ }^{\mathrm{a}} 0,2,3,5$ y $7 ;{ }^{\mathrm{b}} 0,1,2$ y $4 ;{ }^{\mathrm{c}} 0,3,5$ y 7.

En cuanto a la calificación global (Tabla 4), todos los tratamientos recibieron calificaciones desde 0 (Muy bueno), hasta 5 (Muy malo). La prueba de Tukey indicó que la mejor calificación la obtuvo el ensilaje con pasto al corte de 20 días (media de 2,29 - Bueno), mientras que el menor calificado fue el de 13 días (media de 3,54 - Regular).

Tabla 4. Calificación global del ensilaje de pasto Saboya

Table 4. Overall rating of savoy grass silage

\begin{tabular}{cll}
\hline $\begin{array}{r}\text { Edad } \\
\text { (días) }\end{array}$ & Calificación & Calidad \\
\hline 13 & $3,54 \mathrm{c}$ & Regular \\
20 & $2,29 \mathrm{a}$ & Bueno \\
27 & $3,07 \mathrm{abc}$ & Regular \\
34 & $2,68 \mathrm{ab}$ & Bueno \\
41 & $3,43 \mathrm{bc}$ & Regular \\
\hline
\end{tabular}

Nota: Los resultados con letras diferentes, indican diferencia significativa entre los tratamientos. 


\subsection{Composición química y pH del ensilaje de pasto Saboya}

Los resultados encontrados en esta investigación, sobre composición química y pH del ensilaje, se detallan en la Tabla 5. El contenido de MS aumentó con la edad, variando de 13,22\% hasta 22,41\%, a las edades de 13 y 34 días, respectivamente, con una diferencia estadísticamente significativa entre el tratamiento de 13 días y los tratamientos de 34 y 41 días. La PB disminuyó con la edad, pasando de 7,12 \% a los 13 días hasta $5,53 \%$ a los 41 días, presentando una diferencia estadística entre el tratamiento a 41 días con respecto al resto, al ser el pasto más maduro.

El EE presentó un promedio de 1,97 \% a los 13 días de rebrote, una elevación máxima de 2,69 \% a los 27 días y un descenso hasta $2,2 \%$ a los 41 días, encontrándose diferencias significativas entre el ensilaje de 27 días de rebrote con respecto a los de 13, 20 y 34 días, pero no con el de 41 días. Mientras que la FB aumentó con la edad, existiendo diferencias significativas solamente en el pasto más joven de 13 días. Respecto a las cenizas, la edad no afectó significativamente su contenido.

$\mathrm{Al}$ analizar los extractos no nitrogenados (ENN), el análisis de varianza determinó que éstos tienden a disminuir con la edad hasta los 27 días con un leve aumento a los 34 y 41 días. Se estableció una diferencia significativa entre el ensilaje de 13 días con respecto a los de 27, 34 y 41 días, pero es similar con respecto al de 20 días. En lo que se refiere al pH, existe una tendencia a disminuir con la edad, excepto en el tratamiento de 35 días, encontrándose, por lo tanto, diferencias significativas entre este tratamiento y el de 41 días.

Tabla 5. Análisis bromatológico del ensilaje de pasto Saboya

Table 5. Bromatological analysis of savoy grass silage

\begin{tabular}{clllllll}
\hline $\begin{array}{c}\text { Edad } \\
\text { (días) }\end{array}$ & MS (\%) & PB (\%) & EE (\%) & FB (\%) & Ceniza (\%) & ENN (\%) & pH \\
\hline 13 & $13,23 \mathrm{~b}$ & $7,12 \mathrm{a}$ & $1,98 \mathrm{~b}$ & $27,52 \mathrm{~b}$ & 12,96 & $50,42 \mathrm{a}$ & $6,10 \mathrm{ab}$ \\
20 & $16,08 \mathrm{ab}$ & $7,30 \mathrm{a}$ & $2,15 \mathrm{~b}$ & $30,27 \mathrm{ab}$ & 11,76 & $48,53 \mathrm{ab}$ & $6,35 \mathrm{bc}$ \\
27 & $18,89 \mathrm{ab}$ & $8,25 \mathrm{a}$ & $2,70 \mathrm{a}$ & $32,89 \mathrm{a}$ & 13,3 & $42,86 \mathrm{c}$ & $6,09 \mathrm{ab}$ \\
34 & $22,42 \mathrm{a}$ & $7,38 \mathrm{a}$ & $2,09 \mathrm{~b}$ & $33,28 \mathrm{a}$ & 12,61 & $44,64 \mathrm{bc}$ & $6,55 \mathrm{c}$ \\
41 & $20,23 \mathrm{a}$ & $5,53 \mathrm{~b}$ & $2,20 \mathrm{ab}$ & $33,20 \mathrm{a}$ & 12,93 & $45,00 \mathrm{bc}$ & $5,79 \mathrm{a}$ \\
$\boldsymbol{p}$ & $0,0043^{*}$ & $0,0002^{*}$ & $0,0098^{*}$ & $0,0003^{*}$ & 0,1609 & $0,0007^{*}$ & $0,0018^{*}$ \\
\hline
\end{tabular}

*Diferencias significativas

Nota: Resultados con la misma letra no tienen diferencia significativa.

\subsection{Paredes celulares del ensilaje de pasto Saboya}

Al finalizar este estudio, la fibra detergente neutro (FDN) aumentó con la edad y marcó dos grupos bien definidos por la diferencia estadísticamente significativa: el primero conformado por los tratamientos de 13 a 27 días (56,08 \% a 56,67 \%) y el segundo por los de 34 y 41 días $(63,87 \%$ y 64,35\%). En cambio, la fibra detergente ácido (FDA) no presentó diferencias significativas entre tratamientos, mientras que la lignina ácido detergente (LAD), aumentó con la edad, presentando una media de 9,83\% a los 13 días y de $13,18 \%$ a los 41 días, existiendo diferencias significativas entre los tratamientos de 13 y 20 días con respecto a los de 34 y 41 días; el tratamiento de 27 días no tuvo diferencia con ningún otro tratamiento (Tabla 6).

Tabla 6. Composición de paredes celulares del ensilaje de pasto Saboya

Table 6. Cell wall composition of savoy grass silage

\begin{tabular}{cllc}
\hline Edad (días) & FDN (\%) & FDA (\%) & LAD (\%) \\
\hline 13 & $56,40 \mathrm{a}$ & 48,19 & $9,83 \mathrm{a}$ \\
20 & $56,08 \mathrm{a}$ & 48,13 & $10,09 \mathrm{a}$ \\
27 & $56,67 \mathrm{a}$ & 47,26 & $11,41 \mathrm{ab}$ \\
34 & $63,87 \mathrm{~b}$ & 48,19 & $12,32 \mathrm{~b}$ \\
41 & $64,35 \mathrm{~b}$ & 52,33 & $13,18 \mathrm{~b}$ \\
$\boldsymbol{p}$ & $0,0000^{*}$ & 0,0964 & $0,0001^{*}$
\end{tabular}

Nota: *Significación estadística de $p$ al $5 \%$

Los resultados que tienen la misma letra no tienen diferencia significativa. 


\section{Discusión}

\subsection{Rendimiento de materia seca y relación H/T del pasto Saboya}

Homen et al. (2010) al analizar el rendimiento de MS de pasto Saboya, a diferentes edades de corte y en 4 periodos del año, obtuvieron resultados similares a los obtenidos en el período de mínima precipitación, con 0,48; 1,28; 2,16; y 3,7 toneladas de MS ha ${ }^{-1}$ corte $^{-1}$ a las edades de 21, 28, 35 y 42 días. Por su parte, Ramírez Reynoso et al. (2009) analizaron el rendimiento de MS del mismo pasto a las edades de 21, 35 y 42 días, obteniendo resultados un poco más elevados: 2,4; 3,4 y 3,7 toneladas, respectivamente. Así mismo, Verdecia et al. (2008) indican que el rendimiento en MS aumenta con la edad de la planta, encontrándose valores mayores a los 105 días con $\left(12,7 \mathrm{t} \mathrm{ha}^{-1}\right.$ corte $\left.^{-1}\right)$, aunque el mismo autor señala que las plantas se desarrollaron en condiciones específicas, por ejemplo, en los meses de mayor intensidad luminosa, temperatura y precipitaciones, en contraste con los meses opuestos.

Respecto a la relación $\mathrm{H} / \mathrm{T}$, los resultados de este estudio son aceptables al compararlos con otros estudios, ya que la relación $\mathrm{H} / \mathrm{T}$ varía de acuerdo con la ubicación y edad, teniendo variaciones desde 1 hasta 4,8 . Izurieta Pincay (2015) reporta una relación H/T de 4,1; 4,8; 3,2; y 2,2 para las edades de 28, 42, 56 y 70 días, respectivamente del pasto Saboya, durante la época seca en Quevedo-Ecuador. Ramírez Reynoso et al., (2009) obtuvieron una relación H/T del pasto Saboya en época seca de 3,1; 2,1; y 1,6 a edades de 21, 35 y 49 días respectivamente. Homen et al. (2010) al estudiar el mismo pasto durante la época seca, reportaron resultados de aproximadamente 1,$4 ; 2,9 ; 2,3 ; 1$ y 1,2 a una edad del pasto de $21,28,35,42$ y 49 días respectivamente. Verdecia et al. (2008) indican que la proporción H/T disminuye con la edad, siendo mejor a los 30 días con 11,62 \%. En otro estudio se indica que el pasto Saboya, cosechado después de los 55 días de rebrote, dispone de una población importante de Lactobacillus plantarum, lo cual es importante para que se dé una adecuada fermentación y aumento de la recuperación de MS (Santos et al., 2014).

\subsection{Composición química y $\mathrm{pH}$ del ensilaje de pasto Saboya}

Los resultados obtenidos en esta investigación son más elevados que los obtenidos por Vasconcelos et al. (2009) en Brasil, quien determinó 19,75; 19,91; 20,5 y 25,1 \% a los 35, 45, 55 y 65 días, respectivamente. Loures et al. (2005a) reportan un valor de $26,35 \%$ de MS en ensilaje de la variedad Tanzania a 45 días de edad con 5 horas de presecado y 15,65 \% de MS sin presecado. En otro estudio, los mismos autores reportan valores de 24,2 y 20,6\% de MS en ensilaje de 45 días de edad con y sin presecado, respectivamente (Loures et al., 2005b). Estas diferencias entre estudios podrían deberse a la implementación o no de presecado y su duración. Por su parte, Wascheck et al. (2008) reportan los cambios de MS del pasto Saboya de 70 días de edad, sometido a 0, 2, 3 y 5 horas de presecado, con resultados de 25,16; 29,57; 29,83 y 33,02\% de MS, respectivamente.

Respecto a la PB, los resultados obtenidos son similares a los presentados por Vasconcelos et al. (2009) quienes obtuvieron 7,69; 7,52; 7,71 y 5,26 \% de PB a edades de 35, 45, 55 y 65 días, respectivamente. Loures et al. (2005a) reportan un valor de $6,85 \%$ de PB en ensilaje del pasto a 45 días de edad, mientras que González Albarracín (2013) reporta valores mayores, al incluir contenido ruminal en el ensilaje de pasto de 45 días de edad con una PB de 8,21 \%. Así mismo, los resultados obtenidos no coinciden con los indicados por Dele et al. (2013), en donde el contenido de proteína cruda fue mucho más alto $(14,44 \%)$, sin embargo, en este estudio se usó pasto Saboya, cáscara de yuca y residuos de anacardo en diferentes períodos de ensilaje. Verdecia et al. (2008) indican que la proteína disminuye con la edad, siendo mejor a los 30 días, debido a la reducción de la síntesis de compuestos proteicos por la disminución de la cantidad de hojas del pasto.

En lo concerniente al EE, se obtuvo la misma tendencia en el estudio de Villota González (2012), aunque con valores un poco menores: $1,17 \%, 1,62 \%$ y $1,06 \%$ a los 28,35 y 42 días de rebrote, respectivamente. Sin embargo, Baldelomar (2005) obtuvo resultados de $1,4 \%$ y 5,5 \% a los 20 y 40 días, mientras que González Albarracín (2013) informa valores mayores al adicionar contenido ruminal al ensilaje de pasto Saboya, obteniendo resultados de $6,17 \%$ a los 45 días de rebrote.

En cuanto a la FB, González Albarracín (2013) reportó un crecimiento lineal en esta variable, pero con valores mayores, pasando de $38 \%$ a 40,4 \% de FB a los 45 y 60 días de rebrote, respectivamente, diferencia que podría estar relacionada a la inclusión de contenido ruminal que fue adicionado en su estudio. Mientras tanto Baldelomar (2005) obtuvieron valores similares a los de esta investigación, con 30 hasta 31,9 \% de FB a las edades de 20 y 40 días, respectivamente. El contenido de cenizas obtenido difiere con lo expuesto por Villota González (2012) y González Albarracín (2013), quienes sostienen que el mismo aumenta con la edad. Resultados parecidos de ENN reportó Villota González (2012) al obtener 40,87 \% a los 28 días, con una leve 
disminución a 40,29 \% a los 35 días y aumento a 40,86 \% a los 42 días. Por otra parte, González Albarracín (2013) reporta valores menores en el ensilaje de pasto Saboya con inclusión de contenido ruminal, siendo de $35,25 \%$ a los 45 días y $34,66 \%$ a los 60 días de edad.

Respecto al pH, Rigueira et al., (2013) reportaron valores de 4,77 en el pasto de 70 días, mientras que Santos et al. (2014) indicaron 4,74 en el análisis a 50 días de rebrote. Pholsen et al., (2016) reportaron un aumento 4,67 a 5,16 de $\mathrm{pH}$ al presecar el pasto Saboya de 60 días, así como Loures et al. (2005a) observaron el mismo efecto al aumentar de 6,07 a 6,26 con un presecado de 5 horas en pasto Saboya de 45 días. Wascheck et al. (2008) determinaron que cuanto mayor sea la exposición de pasto al presecado, mayor es el valor de $\mathrm{pH}$ al observar los siguientes resultados: 4,$3 ; 4,8 ; 5,5$ y 6 ante una exposición de $0,2,3$ y 5 horas de presecado, respectivamente. González Albarracín (2013) analizó el pH en ensilaje de pasto Saboya de 45 y 60 días de edad con la inclusión de contenido ruminal, obteniendo un $\mathrm{pH}$ de 5,57 y 5,35, respectivamente; con base en la bibliografía revisada, el pH aumenta con la exposición al presecado y disminuye con la inclusión de melaza (Zhang et al., 2010).

\subsection{Paredes celulares del ensilaje de pasto Saboya}

Los resultados obtenidos de FDN son similares a los conseguidos por Vasconcelos et al. (2009), quienes obtuvieron valores 56,$73 ; 58,73 ; 63,12$ y $66,64 \%$ en ensilaje de pasto Saboya a las edades de 35, 45, 55, y 65 días, respectivamente. Por su parte, Villota González (2012) reportó valores un poco más altos en pasto Saboya fresco ( 71,$98 ; 72,3$ y $71,95 \%$ a las edades de 28,35 y 42 días, respectivamente) y con una tendencia más bien cuadrática, pero sin diferencias significativas.

Los datos de FDA obtenidos son análogos a los conseguidos por Villota González (2012), quien al analizar pasto Saboya fresco a los 28, 35 y 42 días, alcanzó 40,41; 41,36 y 40,41 \% de FDA, respectivamente. Mientras tanto, Loures et al. (2005b) obtuvieron porcentajes mayores, llegando a 45,2 y $49 \%$ de FDA en ensilaje con y sin presecado a los 45 días. Verdecia et al. (2008) indican que al aumentar la edad de los pastos, existe un aumento significativo de FDA y LAD, debido a una mayor síntesis de carbohidratos estructurales, lo que reduce la calidad del pasto.

\section{Conclusiones}

$\mathrm{Al}$ analizarse las características de las diferentes edades de corte, se concluye que el mejor ensilaje fue el de 27 días, mientras que en rendimiento el de 41 días, sin embargo, ninguno alcanzó un nivel óptimo de fermentación ( $\mathrm{pH}$, lignificación relación tallo/hoja). Así mismo, el mayor rendimiento de biomasa seca, por secado al sol y por corte, se obtuvo a los 41 días de edad del pasto y la relación T/H a la edad de 27 días. En la calificación global de los ensilajes se determina que los pastos de 20 y 34 días de edad son de buena calidad y los otros son de calidad regular. En el análisis bromatológico y de paredes celulares se determinó que la PB y los ENN disminuyeron con la edad, en tanto que la MS, la FB, la FDN y la LAD aumentaron, afectando la calidad del mismo. El pH aparentemente disminuyó con la edad, pero en ningún tratamiento alcanzó niveles óptimos. El ensilaje realizado con pasto de 27 días de edad es el que cuenta con un rendimiento aceptable de MS sin perjudicar a la calidad nutricional, siendo el de mayor PB y aceptable lignificación, además de presentar el segundo pH más bajo y contar con la mayor relación hoja/tallo, por lo que se debería buscar mejorar la fermentación a esta edad.

\section{Agradecimiento}

Agradecimiento a la Hacienda el Triunfo y su personal por su apertura y ayuda incondicional.

\section{Contribuciones de los autores}

- Katy Derichs: conceptualización, investigación, metodología, recursos, redacción - borrador original, revisión y edición.

- Jorge Mosquera: conceptualización, investigación, metodología, supervisión.

- $\quad$ Lenin Javier Ron-Garrido: Curación de datos, análisis formal.

- Byron Puga-Torres: validación, redacción, revisión y edición.

- Francisco de la Cueva: conceptualización, investigación, metodología, administración del proyecto, supervisión. 


\section{Referencias}

Baldelomar, E. (2005). Producción y análisis bromatológico de tres gramíneas tropicales (B. decumbens, Panicum maximun cv., Tanzania y cv. Gatton). Universidad Autónoma Gabriél René Moreno. http://www.sidalc.net/ cgi-bin/wxis.exe/?IsisScript $=$ cidab.xis\&method $=$ post $\&$ formato $=2 \&$ cantidad $=1 \&$ expresion $=\mathrm{mfn}=013092$

Batello, C. (2000). Uso del ensilaje en el trópico privilegiando opciones para pequeños campesinos. FAO. http://www.fao.org/3/x8486s/x8486s.pdf

Del Pezo, P. (2015). La sequía acorrala a los ganaderos de Santa Elena. El Universo. https://www.eluniverso. com/noticias/2015/03/03/nota/4615321/sequia-acorrala-ganaderos-santa-elena/

Dele, P., Jolaosho, A, Arigbede, O., Ojo, V., Amole, T., Okukenu, O., \& Akinyemi, B. (2013). Chemical composition and in vitro gas production of silage from guinea grass, cassava peel and cashew apple waste at different periods of ensilage. Pakistan Journal of Biological Sciences, 16(23):1801-1805. https://doi. org/10.3923/pjbs.2013.1801.1805

González Albarracín, L. W. (2013). Evaluación de la composición nutricional de microsilos de King Grass Pennisetum purpureum y pasto Saboya Panicum maximun Jacq, en dos estados de madurez con 25\% de contenido ruminal de bovinos faenados en el camal municipal del canton Quevedo. Universidad Técnica de Cotopaxi.

Homen, M., Entrena, I., Arriojas, L., \& Ramia, M. (2010). Biomasa y valor nutritivo del pasto Guinea Megathyrsus maximus (Jacq.) B. K. Simon y S. W. L. Jacobs. 'Gamelote' en diferentes períodos estado Miranda. Zootecnia Tropical, 28(2), 255-265. http://ve.scielo.org/scielo. php?pid=S0798-72692010000200011\&script=sci_abstract

Instituto Nacional de Estadísticas y Censos [INEC]. (2014). Encuesta de superficie y producción agropecuaria continua ESPAC 2014. INEC. https://www.ecuadorencifras.gob.ec/documentos/web-inec/Estadisticas agropecuarias/espac/espac_2014-2015/2014/Informe\%20ejecutivo\%20ESPAC\%202014.pdf

Instituto Nacional de Estadísticas y Censos [INEC]. (2019). Encuesta de superficie y producción agropecuaria continua (ESPAC). 2018. INEC. https://www.ecuadorencifras.gob.ec/documentos/web-inec/Estadisticas agropecuarias/espac/espac-2018/Presentacion de principales resultados.pdf

Instituto Nacional de Estadísticas y Censos [INEC]. (2021). Encuesta de Superficie y Producción Agropecuaria Continua 2020. INEC. https://www.ecuadorencifras.gob.ec/documentos/web-inec/Estadisticas_agropecuarias/espac/espac-2020/Presentacion ESPAC 2020.pdf

Izurieta Pincay, W. R. (2015). Determinación del rendimiento forrajero y valor nutritivo del pasto Saboya (Panicum maximum Jacq.) sujeto a cuatro frecuencias de corte durante la época seca en Quevedo. Escuela Politécnica del Litoral.

Junges, D. (2010). Avaliação sensorial da qualidade de forragem conservada. Universidade Federal Do Paraná, Departamento de Zootecnia Centro de Pesquisa em Forragicultura (CPFOR). https://www.ensilagem.com. br/avaliacao-sensorial-da-qualidade-de-forragem-conservada/

Lara, L. A., Santos, M. F., Jank, L., Chiari, L., Vilela, M. de M., Amadeu, R. R., dos Santos, J. P. R., Pereira, G. da S., Zeng, Z.-B., \& García, A. A. F. (2019). Genomic selection with allele dosage in Panicum maximum Jacq. G3 Genes|Genomes|Genetics, 9(8), 2463-2475. https://doi.org/10.1534/g3.118.200986

Loures, D. R. S., Nussio, L. G., Paziani, S. de F., Pedroso, A. de F., Mari, L. J., Ribeiro, J. L., Zopollatto, M., Schmidt, P., Junqueira, M. C., Packer, I. U., \& Campos, F. P. de. (2005a). Composição bromatológica e produção de efluente de silagens de capim-tanzânia sob efeitos do emurchecimento, do tamanho de partícula e do uso de aditivos biológicos. Revista Brasileira de Zootecnia, 34(3), 726-735. https://doi.org/10.1590/ S1516-35982005000300003

Loures, D. R. S., Nussio, L. G., Paziani, S. de F., Pedroso, A. de F., Mari, L. J., Ribeiro, J. L., Zopollatto, M., Schmidt, P., Junqueira, M. C., Packer, I. U., \& Campos, F. P. de. (2005b). Efeito de enzimas fibrolíticas e do teor de matéria seca em silagens de capim-tanzânia sobre os parâmetros ruminais, o comportamento ingestivo e a digestão de nutrientes, em bovinos. Revista Brasileira de Zootecnia, 34(3), 736-745. https:// doi.org/10.1590/S1516-35982005000300004

Machado, F. (2016). La sequía causa problemas en Cuenca, Latacunga, Manta y en la frontera. El Telégrafo. https://www.eltelegrafo.com.ec/noticias/ecuador/1/la-sequia-causa-problemas-en-cuenca-latacunga-manta-y-en-la-frontera

Mannetje, L. (2005). Introducción a la conferencia sobre el uso del ensilaje en el trópico. En: L- 't Mannetje, Uso del Ensilaje en el Trópico Privilegiando Opciones para Pequeños Campesinos (pp. 1-4). FAO. http:// www.fao.org/3/x8486s/x8486s03.htm 
Montenegro, L. (2019). Características de fermentación y nutritivas de ensilajes de forrajes tropicales con diferentes niveles de inclusión de subproductos agroindustriales. Universidad de Córdoba.

Núñez Delgado, J., Ñaupari Vasquez, J., \& Flores Mariazza, E. (2019). Comportamiento nutricional y perfil alimentario de la producción lechera en pastos cultivados (Panicum maximum Jacq). Revista de Investigaciones Veterinarias del Perú, 30(1), 178-192. https://doi.org/10.15381/rivep.v30i1.15681

Nussbaum, H., Weißbach, F., Staudacher, W., von Borstel, U., Groß, F., Seibold, R., \& Rieder, J. B. (2004). Grobfutterbewertung Teil A - DLGSchlüssel zur Bewertung von Grünfutter, Silage und Heu mit Hilfe der Sinnenprüfung. DLG - Deutsche Landwirtschafts-Gesellschaft e. V., -DLG. https://www.gruenland-online.de/medien/grobfutterbewertunga.pdf

Nussio, L. G., Paziani, S. F., \& Nussio, C. M. B. (2002). Ensilagem de capins tropicais. Reunião Anual da Sociedade Brasileira de Zootecnia, p. 60-99. https://ainfo.cnptia.embrapa.br/digital/bitstream/CPPSE/14080/1/ PROCICMBN2002.00031.PDF

Olivera Viciedo, D., de Mello Prado, R., Martínez, C. A., Habermann, E., \& de Cássia Piccolo, M. (2019). Shortterm warming and water stress affect Panicum maximum Jacq. stoichiometric homeostasis and biomass production. Science of The Total Environment, 681, 267-274. https://doi.org/10.1016/j.scitotenv.2019.05.108

Ohmomo, S., Tanaka, O., Kitamoto, H. K., \& Cai, Y. (2002). Silage and Microbial Performance, Old Story but New Problems. Japan Agricultural Research Quarterly: JARQ, 36(2), 59-71. https://doi.org/10.6090/ jarq.36.59

Parvin, S., \& Nishino, N. (2009). Bacterial community associated with ensilage process of wilted guinea grass. Journal of Applied Microbiology, 107, 2029-2036. https://doi.org/10.1111/j.1365-2672.2009.04391.x

Pessim, C., Pagliarini, M. S., Silva, N., \& Jank, L. (2015). Chromosome stickiness impairs meiosis and influences reproductive success in Panicum maximum (Poaceae) hybrid plants. Genetics and Molecular Research: GMR, 14(2), 4195-4202. https://doi.org/10.4238/2015.April.28.2

Pholsen, S., Khota, W., Pang, H., Higgs, D., \& Cai, Y. (2016). Characterization and application of lactic acid bacteria for tropical silage preparation. Animal Science Journal, 87(10), 1202-1211. https://doi.org/10.1111/ asj. 12534

Queiroz, O. C. M., Ogunade, I. M., Weinberg, Z., \& Adesogan, A. T. (2018). Silage review: Foodborne pathogens in silage and their mitigation by silage additives. Journal of Dairy Science, 101(5), 4132-4142. https:// doi.org/10.3168/jds.2017-13901

Ramírez Reynoso, O., Hernández Garay, A., Silva, S. C. da, Pérez Pérez, J., Enríquez Quiroz, J. F., Quero Carrillo, A. R., Herrera Haro, J. G., \& Cervantes Núñez, A. (2009). Herbage accumulation, growth and structural characteristics of Mombasa grass (Panicum maximum Jacq) harvested at different citting intervals. Técnica pecuaria en México, 47(2), 203-213. https://www.redalyc.org/pdf/613/61312116008.pdf

Rigueira, J. P. S., Pereira, O. G., Ribeiro, K. G., Mantovani, H. C., \& Agarussi, M. C. N. (2013). The chemical composition, fermentation profile, and microbial populations in tropical grass silages. Revista Brasileira de Zootecnia, 42(9), 612-621. https://doi.org/10.1590/S1516-35982013000900002

Rodríguez López, M. (2009). Rendimiento y valor nutricional del pasto Panicum maximun CV. Mombaza a diferentes edades y alturas de corte. Instituto Tecnológico de Costa Rica.

Santos, E. M., Pereira, O. G., García, R., Ferreira, C. L. L. F., Oliveira, J. S., \& Silva, T. C. (2014). Effect of regrowth interval and a microbial inoculant on the fermentation profile and dry matter recovery of guinea grass silages. Journal of Dairy Science, 97(7), 4423-4432. https://doi.org/10.3168/jds.2013-7634

Vasconcelos, W. A., Santos, E. M., Zanine, A. de M., Pinto Ferreira, T., Lima Costa, W., Edvan Loiola, R., \& Pereira Gomes, O. (2009). Valor nutritivo de silagens de capim-mombaça (Panicum maximum Jacq.) colhido em função de idades de rebrotação. Revista Brasileira de Saúde e Produção Animal, 10(4), 874-884.

Venegas, S. (2017). La historia en la ganadería del Ecuador. El Agropecuario, 540, 10-12.

Verdecia, D., Ramírez, J., Leonard, I., Pascual, Y., \& López, Y. (2008). Rendimiento y componentes del valor nutritivo del Panicum maximum cv. Tanzania (Yiel and component of the nutritive value of the Panicum maximum c.v Tanzania). REDVET. Revista Electrónica de Veterinaria, IX(5), 1-9. https://www.redalyc. org/pdf/636/63611397008.pdf

Villota González, L. (2012). Determinación de la composición química y degradabilidad ruminal in situ de dos componentes del pasto Saboya (Panicum maximum Jacq.) cosechado a tres edades de rebrote en el recinto "Chaflu" de la provincia de Esmeraldas. Universidad Tecnológica Equinoccial.

Wascheck, R. de C., Moreira, P. C., Neto, J. F. F., Costa, D. S., Dutra, A. R., Moreira, L., Campos, R. M., Laforga, C. de S., Rezende, P. L. de P., \& Rabelo, N. A. (2008). Características da silagem de capim 
colonião (Panicum maximum, Jacq) submetido a quatro tempos de emurchecimento pré-ensilagem. Revista EVS - Revista de Ciências Ambientais e Saúde, 35(3), 385-399. http://dx.doi.org/10.18224/est.v35i3.740

Wedow, J. M., Yendrek, C. R., Mello, T. R., Creste, S., Martínez, C. A., \& Ainsworth, E. A. (2019). Metabolite and transcript profiling of Guinea grass (Panicum maximum Jacq) response to elevated $\left[\mathrm{CO}_{2}\right]$ and temperature. Metabolomics, 15(4), 51. https://doi.org/10.1007/s11306-019-1511-8

Zhang, J.-G., Kawamoto, H., \& Cai, Y.-M. (2010). Relationships between the addition rates of cellulase or glucose and silage fermentation at different temperatures. Animal Science Journal, 81(3), 325-330. https:// doi.org/10.1111/J.1740-0929.2010.00745.X 